\title{
Challenges in thermal noise for 3rd generation of gravitational wave detectors
}

\author{
Ronny Nawrodt • Sheila Rowan • Jim Hough • \\ Michele Punturo • Fulvio Ricci · Jean-Yves Vinet
}

Received: 8 October 2009 / Accepted: 7 June 2010 / Published online: 9 August 2010

(C) Springer Science+Business Media, LLC 2010

\begin{abstract}
Various noise sources limit the sensitivity of current interferometric gravitational wave detectors, including seismic noise, thermal noise of the optical components and suspension elements and photon shot noise. Plans are in place for a suite of hardware upgrades which should increase the sensitivity of these detectors by reducing the various noise sources. With these designs for 2 nd generation detectors mature, techniques for further improvement of detector sensitivity by a factor of approximately 10 are under study. A particular challenge is the reduction of the thermal noise associated with the interferometer mirrors and their suspensions. We review the current status of research on thermal noise in interferometric gravitational wave detectors. Aspects of possible techniques for use in future '3rd generation detectors' such as cryogenics and diffractive optics are discussed.
\end{abstract}

Keywords Gravitational waves · Thermal noise

R. Nawrodt $(\varangle) \cdot$ S. Rowan · J. Hough

Institute for Gravitational Research, University of Glasgow, Glasgow G12 8QQ, Scotland, UK

e-mail: r.nawrodt@physics.gla.ac.uk

S. Rowan

e-mail: s.rowan@physics.gla.ac.uk

M. Punturo

Instituto Nazionale di Fisica Nucleare, 06100 Perugia, Italy

F. Ricci

Dipartimento di Fisica, Università di Roma La Sapienza, Rome, Italy

J.-Y. Vinet

Observatoire de la Cote d'Azur, Bd de l'Observatoire, 06304 Nice Cedex 4, France 


\section{Introduction}

Upcoming generations of gravitational wave detectors like Advanced Virgo [1] and Advanced LIGO [2] are expected to have sensitivities limited by a variety of noise sources such as seismic noise, quantum noise and thermal noise of the optical components and their suspension elements [3]. Looking beyond these 'Advanced' designs, a design goal for future ' 3 rd generation' gravitational wave interferometers is to achieve a sensitivity approximately 10 times better than Advanced instruments over a detection band from $<10 \mathrm{~Hz}$ up to $10 \mathrm{kHz}[4,5]$. A conceptual design study for such a potential 3rd generation detector in Europe - the Einstein Telescope (ET) — started in spring 2008 [5]. Groups from several European countries are working towards developing a first design of such a detector, proposing a combination of currently available techniques and upcoming novel ideas, aiming at a sensitivity level of approximately that shown in Fig. 1. This forms a significant scientific challenge.

A range of novel experimental techniques are under investigation as possible routes towards achieving this goal, such as the use of very high laser powers (up to MW [6]), squeezed light [7-9], the use of diffractive optics [10-13], non-Gaussian laser beam profiles [14-16] and the use of cryogenic techniques [17]. The requirements for very low seismically induced noise (especially gravity gradient noise) make siting a detector underground an option of particular interest $[18,19]$.

Simple extensions of current technologies seem unlikely to provide a suitable path towards desired sensitivities. In some areas, technological limits start to become significant, e.g. in achieving the necessary specifications on mirror curvatures or substrate diameters. In addition, techniques like the use of very high laser powers impose technical constraints (such as the need to handle high heat loads on the mirrors) that conflict with the use of other techniques, like cryogenics for reducing thermal noise (where cooling the optics to temperature of $20 \mathrm{~K}$ or less is desirable). Thus, some care must be exercised in the choice and combination of the array of techniques available for use.

Fig. 1 Comparison of the sensitivity curves of 1 st, 2nd and 3rd generation gravitational wave detectors [6]

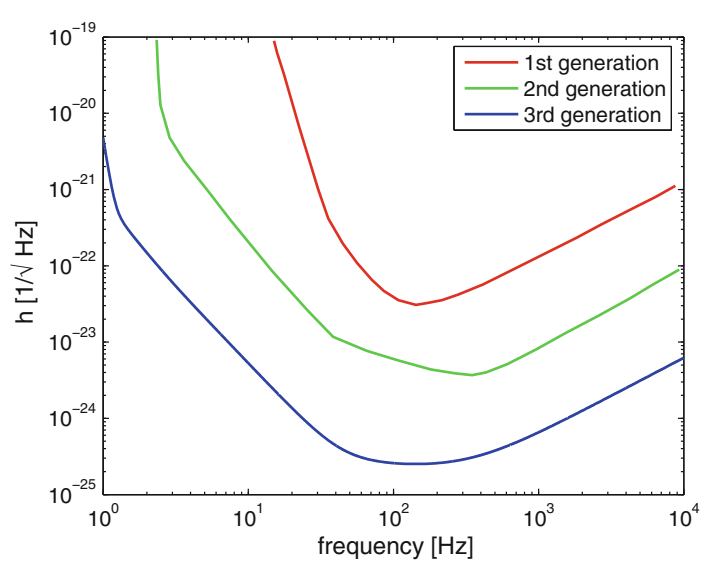


The 'xylophone' concept [20,21] of dividing the frequency range into two or more bands to be targeted by detectors of different technical design is of interest here. A detector with a goal of good sensitivity at higher frequencies might be operated at or close to room temperature, and be illuminated by high laser powers. A low frequency instrument could instead support more moderate laser power but employ cryogenic techniques to reduce the thermal noise of the optical components. The experimental implementation of such a scheme would employ additional optics but might use common infrastructure (vacuum tubes, underground tunnels).

Particularly important is the thermal noise which is associated with the ion-beamsputtered dielectric mirror coatings. These coatings are applied to the optic substrates in order to create mirrors of high-reflectivity at 1,064 nm [22-24]. Thus, a reduction of the thermal noise associated with the coatings is a research topic of high priority.

This article summarises significant aspects of the current status of research on thermal noise in optical components and suspension elements for gravitational wave interferometers. An overview of the calculation techniques typically used in estimating mirror and suspension thermal noise is given, followed by a discussion of the levels of thermal noise anticipated in advanced detector designs. Subsequently, challenges in reducing thermal noise towards the levels desired for 3rd generation detectors are discussed and consideration is given to the choice of appropriate materials for use in the optical substrates, coatings and suspensions elements of such a detector. The thermal noise of a simplified design of 3rd generation detector is calculated and an overview of novel ideas of how to further reduce thermal noise is presented in Sect. 5.

\section{Thermal noise of optical components}

The thermal noise of the test-masses and suspension in interferometric gravitational wave detectors arises from the thermal energy of their atoms and molecules which have a thermal energy of $k_{\mathrm{B}} T / 2$ per degree of freedom. Thermal noise is manifest typically in two forms. The first is Brownian thermal noise [25], where thermal fluctuations couple to the surface displacement of the optical component via the effects of spatially distributed sources of mechanical friction. The second arises from the statistical fluctuations of the temperature of a system which are caused by random heat fluxes. These temperature fluctuations couple to the local displacement of the surface by means of the linear expansion coefficient. In the systems considered here this may be present as thermo-elastic noise [26,27]. In transmissive optics the temperature fluctuations additionally cause local random changes in the refraction index by means of its temperature dependence. This type of noise is called thermo-refractive noise [28].

A consequence of the fluctuation-dissipation theorem is that any system in which energy is dissipated must exhibit fluctuations of its measurable (energy dependent) parameters $[29,30]$.

In a mechanical resonant system like the one considered here the power spectral density of a fluctuating driving force $S_{\mathrm{F}}(f, T)$ is a function of the real part of the 
mechanical admittance $\operatorname{Re}[Y(f)]$

$$
S_{\mathrm{F}}(f, T)=\frac{k_{\mathrm{B}} T}{\pi^{2} f^{2}} \operatorname{Re}[Y(f)],
$$

where $k_{\mathrm{B}}=1.38 \times 10^{-23} \mathrm{~J} / \mathrm{K}$ is Boltzmann's constant, $T$ is the sample temperature and $f$ is frequency.

A commonly used approach in the design and analysis of suspension performance has been to model the resonant modes of test masses and suspensions their suspensions as damped harmonic oscillators, each of some mass $m$ and resonant eigenfrequency $f_{0}$. Applying the fluctuation-dissipation theorem, the power spectral density of thermal displacement noise, $S_{\mathrm{x}}(f, T)$ associated with each mode is given by Saulson [31]

$$
S_{\mathrm{X}}(f, T)=\frac{k_{\mathrm{B}} T f_{0}^{2} \phi(f)}{2 \pi^{3} f m\left[\left(f^{2}-f_{0}^{2}\right)^{2}+f_{0}^{4} \phi^{2}(f)\right]} .
$$

Hence, when choosing materials for the test masses and suspensions of gravitational wave detectors, materials of low mechanical loss $\phi(f, T)$, are highly desirable.

In the model referenced above, the test mass position is determined from laser light reflection from the front of the mirror surface. The measured surface position is determined, in part, by thermally activated displacements, and it is assumed that the displacements from non-resonant motion do not sum coherently [32]. Several authors developed different approaches to calculating thermal noise [33-35] which show that the simple treatment presented above only holds if the off-resonance thermal noise from the various resonant modes is uncorrelated. In current mirror suspensions the distribution of the mechanical loss has been spatially inhomogeneous which leads to correlation between noise from different modes. This situation can cause a significant change in the predicted level of thermal noise compared to that which would be predicted using a simple 'modal' approach $[33,36]$.

\subsection{Bulk thermal noise}

\subsubsection{Bulk Brownian noise}

If the approach of [33] is applied, it can be shown that the amplitude power spectral density $S_{\mathrm{x}}(f, T)$ of Brownian thermal noise associated with a bulk mirror substrate may be given by:

$$
S_{x}^{\text {bulk }}(f, T)=\frac{4 k_{\mathrm{B}} T}{\pi f} \frac{U_{\max }}{F_{0}^{2}} \times \phi_{\text {bulk }}(f) .
$$

$\phi_{\text {bulk }}(f)$ is the mechanical loss factor of the bulk material. $U_{\max }$ is the maximum energy associated with deformation of the substrate occurring when a notional oscillatory force of peak magnitude $F_{0}$ is applied to the front surface of the substrate, where this force has a pressure distribution of the same spatial profile as the laser beam being 
used to sense the test mass displacement. $U_{\max }$ can be obtained analytically in some cases or, more generally, using finite element modeling techniques. $U_{\max }$ is frequency independent if the frequency is much smaller than the first internal resonance of the mirror. This situation is well fulfilled in the case of GW detectors.

If the laser beam diameter is much smaller than the test mass diameter the test mass can be approximated as a semi-infinite half space. The Brownian thermal noise can then be expressed as follows if a homogeneous loss distribution is assumed [33,34]:

$$
S_{x}^{\text {bulk }}(f, T)=\frac{2 k_{\mathrm{B}} T}{\pi^{3 / 2} f} \frac{1-\sigma^{2}}{w Y} \times \phi_{\text {bulk }}(f) .
$$

$\sigma$ is the Poisson ratio and $Y$ the Young's modulus of the sample. $w$ is the beam radius ${ }^{1}$ of the laser used for readout in the interferometer. Equation (4) suggests a strategy for the selection of a test mass material for low noise operation, that of using a material with a high Young's modulus and a low mechanical loss, preferably at cryogenic temperatures.

Several authors have analyzed the influence of the finite shape of the test mass on Brownian thermal noise [34,35]. Liu and Thorne calculated corrections to a semi-infinite half space model that for typical test mass and laser beam sizes are at the level of $10 \%$ thus a semi-infinite half space as above is a reasonable approach for use in initial estimates of thermal noise.

\subsubsection{Bulk thermo-elastic noise}

Thermoelastic noise is associated with a dissipative flow of heat in a material. Thermoelastic damping [37] in the context of thin flexing fibres is an important factor in considering the design of suspension elements, and this will be discussed later [3840]. In 1999, Braginsky et al. [27] have pointed out that temperature fluctuations near the laser beam spot result in random fluctuations of the mirror surface. The thermal fluctuations are coupled to the displacement by means of the coefficient of thermal expansion $\alpha$. This thermo-elastic noise is particularly important for crystalline materials like sapphire which have a large thermal conductivity and a high coefficient of thermal expansion.

For a semi-infinite half space the amplitude power spectral density of thermo-elastic noise is given by Braginsky et al. [27]:

$$
S_{\mathrm{TE}}^{\text {bulk }}(f, T)=\frac{4 k_{\mathrm{B}} T^{2} \alpha^{2}(1+\sigma)^{2} \kappa}{\sqrt{\pi^{5}} \rho^{2} C^{2} w^{3} f^{2}} .
$$

\footnotetext{
1 Throughout this paper we use the radius definition as the distance from the center of the beam where the intensity drops to $1 / e^{2}$ from the maximum.
} 
$\rho$ is the mass density of the substrate, $\kappa$ the thermal conductivity and $C$ the heat capacity. This equation is only valid if the thermal diffusion length [41]

$$
l_{\mathrm{th}}=\sqrt{\frac{\kappa}{\rho C \omega}}
$$

is smaller than the beam radius $w$ (the adiabatic regime). In currently operating gravitational wave detectors this assumption is fulfilled due to the small thermal conductivity of fused silica and the relatively large laser beams used. However the assumption breaks down for example, if materials with a very high thermal conductivity are considered, e.g. crystalline materials at low temperatures as proposed for 3rd generation detectors [17]. If the adiabatic limit is not fulfilled the expected thermo-elastic noise is smaller than predicted from Eq. (5).

A general treatment of thermo-elastic noise is given by Cerdonio et al. [41]:

$$
S_{\mathrm{TE}}^{\text {bulk }}(f, T)=\frac{4}{\sqrt{\pi}} \alpha^{2}(1+\sigma)^{2} \frac{k_{\mathrm{B}} T^{2} w}{\kappa} J[\Omega],
$$

with

$$
J[\Omega]=\sqrt{\frac{2}{\pi^{3}}} \int_{0}^{\infty} d u \int_{-\infty}^{+\infty} d v \frac{u^{3} e^{-u^{2} / 2}}{\left(u^{2}+v^{2}\right)\left[\left(u^{2}+v^{2}\right)^{2}+\Omega^{2}\right]} .
$$

$\Omega=\omega / \omega_{\mathrm{c}}$ is a dimensionless parameter depending on the frequency and the thermal properties of the sample. In the adiabatic limit $(\Omega \gg 1)$ this leads to Eq. (5) again. The relevance of this correction to the expected thermo-elastic noise level in 3rd generation detectors is discussed in Sect. 4.3.

\subsection{Coating thermal noise}

Consideration of the spatial inhomogeneity of the mechanical loss of coated mirrors $[33,36,42]$ highlights the importance of the location of mechanical loss with respect to the position of the laser beam hitting a mirror. If the approach of [33] is applied (see Sect. 2.1.1) it can be seen that dissipation located close to the front mirror surface surface (where the notional force is applied and where most of the deformation that it generates is found) contributes more than dissipation located far away from the front surface. This insight has considerable consequences: all the primary optical components in gravitational wave detectors are coated with a layer formed from highly reflective dielectric multi-layer stacks on the surface whose displacement is probed by the sensing laser beam. Thus, the intrinsic mechanical loss of these layers will strongly contribute to the overall thermal noise of the component making coating thermal noise a critical issue. 


\subsubsection{Coating Brownian noise}

Coating Brownian noise can be calculated using the approach of [33] (see Sect. 2.1.1). A numerical calculation of the maximum deformation energy $U_{\max }$ immediately allows the noise level to be estimated for finite-sized mirrors. In the limit of having laser beams whose diameter is relatively small compared to the substrate diameter (as is the case for commonly used substrate and beam dimensions in gravitational wave interferometers) the coating is assumed to be a thin layer covering a semi-infinite half-space. The Brownian thermal noise contribution of a dielectric multilayer coating can then be expressed as [22]:

$$
S_{\mathrm{x}}^{\text {coat }}(f, T)=\frac{2 k_{\mathrm{B}} T}{\pi^{2} f} \frac{t}{w^{2} Y}\left(\frac{Y^{\prime}}{Y} \phi_{\|}+\frac{Y}{Y^{\prime}} \phi_{\perp}\right) .
$$

$Y$ and $Y^{\prime}$ are the Young's moduli of the substrate and the coating, respectively. $\phi_{\|}$is the loss angle associated with the energy density of strains in a direction parallel to the plane of the coating and $\phi_{\perp}$ is the mechanical loss for the perpendicular case. $t$ is the coating thickness. Equation (9) assumes that the Poisson's ratios of the bulk and the coating are small. A general treatment including the effect of the Poisson ratio can be found in [22].

In the case of $\phi_{\|}=\phi_{\perp}$ the lowest level of Brownian thermal noise occurs if $Y=Y^{\prime}$. This indicates that similar Young's moduli of the substrate and the coating are preferred in order to obtain the lowest possible thermal noise. As discussed in [22] the ring-down experiments used to measure mechanical loss only reveal $\phi_{\| \mid}$. Within this article it is assumed that the loss is independent of the directions of stress and strain and therefore $\phi_{\|}=\phi_{\perp}$ is fulfilled. Coating Brownian noise is therefore dependent on the mechanical loss of the coating material at a given temperature and the ratio between substrate and coating Young's modulus.

For a multi-layer coating (as is typical for gravitational wave mirrors) two general approaches can be applied. First, the coating is assumed to be an effective medium having an averaged mechanical loss, Young's modulus and Poisson ratio. The averaging process is carried out using a weighing procedure [43]:

$$
(X)_{\mathrm{avg}}=\sum_{i=1}^{N} \frac{Y_{i} t_{i}}{\sum_{j=1}^{N} Y_{j} t_{j}} X_{i}
$$

$X$ represents the parameter to be averaged, $N$ is the number of layers, $Y_{i}$ the Young's modulus and $t_{i}$ the thickness of the $i$ th layer. The second approach is to sum all layers of one material up and use a simplified model having less layers but the full geometrical thickness of the coating. Thus, a binary dielectric layer would consist of only two layers having the total thickness of material 1 and 2 each. Both treatments are in good agreement to better than $5 \%$.

A small correction factor for a finite sized test mass was calculated by Somiya and Yamamoto [44]. This correction becomes important in the limit of large beams compared to the substrate diameter. In order to keep clipping losses in the gravitational 
wave detectors small this limit will not be reached during normal operation and thus this correction has only a very small influence on gravitational wave detectors.

\subsubsection{Coating thermo-elastic noise}

The thin dielectric layers of the multilayer coatings also are a source of thermo-elastic thermal noise $[45,46]$. Fejer et al. [46] obtained the following expression for a multilayer coating:

$$
S_{\mathrm{TE}}^{\text {coat }}(f, T)=\frac{8 k_{\mathrm{B}} T^{2}}{\pi^{2} f} \frac{L}{w^{2}} \frac{\alpha_{\mathrm{S}}^{2} C_{\mathrm{F}}}{C_{\mathrm{S}}^{2}}\left(1+\sigma_{\mathrm{S}}\right)^{2} \Delta^{2} g(\omega),
$$

with the index $S$ for substrate and $F$ for the dielectric film. $L$ is the thickness of the dielectric coating. The parameter $\Delta$ is given by:

$$
\Delta=\frac{C_{\mathrm{S}}}{2 \alpha_{\mathrm{S}} C_{\mathrm{F}}}\left(\frac{\alpha}{1-\sigma}\left[\frac{1+\sigma}{1+\sigma_{\mathrm{S}}}+\left(1-2 \sigma_{\mathrm{S}}\right) \frac{Y}{Y_{\mathrm{S}}}\right]\right)_{\mathrm{avg}}-1 .
$$

(...) avg indicates the averaging operator given by:

$$
(X)_{\mathrm{avg}}=\frac{d_{\mathrm{A}}}{d_{\mathrm{A}}+d_{\mathrm{B}}} X_{\mathrm{A}}+\frac{d_{\mathrm{B}}}{d_{\mathrm{A}}+d_{\mathrm{B}}} X_{\mathrm{B}}
$$

The frequency dependence can be described as:

$$
g(\omega)=\operatorname{Im}\left[-\frac{1}{\sqrt{i \omega \tau_{\mathrm{F}}}} \frac{\sinh \sqrt{i \omega \tau_{\mathrm{F}}}}{\cosh \sqrt{i \omega \tau_{\mathrm{F}}}+R \sinh \sqrt{i \omega \tau_{\mathrm{F}}}}\right] .
$$

with $\tau_{\mathrm{F}}=L^{2} / k_{\mathrm{F}} \cdot k_{\mathrm{F}}$ is the thermal diffusivity of the multilayer. The thermal properties are volume averaged as follows:

$$
\begin{aligned}
C_{F} & =(C)_{\mathrm{avg}}, \\
k_{F} & =\frac{\kappa_{\mathrm{F}}}{C_{\mathrm{F}}}, \\
\kappa_{\mathrm{F}}^{-1} & =\left(\kappa^{-1}\right)_{\mathrm{avg}} .
\end{aligned}
$$

$\kappa$ is the thermal conductivity $C$ the specific heat capacity per unit volume of the dielectric material.

Equation (11) assumes again that the adiabatic assumption (see Sect. 2.1.2) is fulfilled. Due to the fact that the dielectric coatings have a low thermal conductivity within the temperature range from 5 to $300 \mathrm{~K}$ the adiabatic assumption is always fulfilled for frequencies, beam and sample sizes considered in gravitational wave detectors.

It can be seen from Eqs. (11) and (12) that the thermo-elastic noise associated with coatings depends on the properties of both the coating and the bulk substrate material. 


\subsubsection{Coating thermo-refractive noise}

Statistical temperature fluctuations in the coating materials are coupled to changes in refraction index. This leads to changes in the optical path length and thus introduces an additional noise source-thermo-refractive noise. The thermo-refractive noise of a coating layer was described by Braginsky et al. [28]:

$$
S_{\mathrm{TR}}^{\text {coat }}(f, T)=\frac{2 k_{\mathrm{B}} T^{2} \beta_{\mathrm{eff}}^{2} \lambda^{2}}{\pi^{3 / 2} \sqrt{\rho C \kappa f} w^{2}} .
$$

$\beta_{\text {eff }}$ is the thermo-refractive factor:

$$
\beta_{\mathrm{eff}}=\frac{\beta_{1} n_{2}^{2}+\beta_{2} n_{1}^{2}}{4\left(n_{1}^{2}-n_{2}^{2}\right)}
$$

with $\beta_{i}=d n_{i} / d T . n_{i}$ is the refraction index of the layer $i$. Equation (19) assumes that the coating thermal expansion coefficient $\alpha_{i}$ can be neglected compared to $\beta_{i}$. A fluctuation dissipation theorem based approach was developed by Levin [47]. Evans et al. [48] calculated the exact solution, with $\beta_{i}$ in Eq. (19) replaced by $\beta_{i}+\alpha_{i} n_{i}$. From [48] it can be seen that thermo-refractive and thermo-elastic noise is correlated and thus the total combined noise can be reduced if the material properties have the correct temperature dependence. This correlation leads to an expression for 'thermo-optic' noise:

$$
S_{\mathrm{TO}}^{\text {coat }}(f, T)=\frac{2}{\pi^{3 / 2}} \frac{k_{\mathrm{B}} T^{2}}{\sqrt{\kappa \rho C f} w^{2}} \Gamma_{\mathrm{tc}}\left(\alpha_{c} t-\beta_{c}^{*} \lambda\right) .
$$

$\Gamma_{\mathrm{tc}}$ is the thick-coating correction factor and $\beta_{c}^{*}$ the effective thermo-refractive constant given in [48]. ${ }^{2}$ This calculation reveals that thermo-optic noise can be reduced if the materials are selected properly. Thus, the effect of thermo-optic noise is further reduced and believed not to be a limitation for 2 nd and possibly even 3rd generation detectors [48].

\subsection{Current status of mirror thermal noise}

The properties of an Advanced LIGO or Virgo-type interferometer are now assumed as a basis from which to review the current status of the mirror thermal noise levels expected from a 2 nd generation detector. Table 1 compiles key values needed for the calculation of the different types of thermal noise discussed. In order to simplify the problem a high-reflective $\lambda / 4$ multilayer stack was assumed including 18 doublets of

\footnotetext{
2 The equations for $\Gamma_{\mathrm{tc}}$ and $\beta_{c}^{*}$ are long and therefore not repeated here again. They can be found in [48]. We also note that thermo-refractive effects clearly also occur when light passes through the bulk of an optical substrate, however in the interferometer topologies considered here, the use of highly reflective cavities in the interferometer arms means that the bulk thermo-refractive noise is at a level much lower than the other thermal noise sources discussed, where effects at the front faces of the substrates are dominant.
} 
Table 1 Overview of key parameters used for thermal noise calculation of an Advanced LIGO like detector

\begin{tabular}{lll}
\hline Property & Value & References \\
\hline Test mass & & \\
Material & Fused silica & {$[50]$} \\
Diameter & $340 \mathrm{~mm}$ & {$[50]$} \\
Thickness & $200 \mathrm{~mm}$ & {$[50]$} \\
Arm lengths & $4 \mathrm{~km}$ & {$[50]$} \\
$\phi_{\text {bulk }}$ & $4 \times 10^{-10}$ & {$[51]$} \\
Optical coating & & \\
Material & $\mathrm{Ti}^{\mathrm{Ta}} \mathrm{O}_{5} / \mathrm{SiO}_{2}$ stacks & \\
Number of $\lambda / 4$ doublets & 18 & \\
$\phi_{\text {tantala }}$ & $4 \times 10^{-4}$ & {$[52]$} \\
$\phi_{\text {silica }}$ & $5 \times 10^{-5}$ & {$[43]$} \\
Laser & & \\
Wavelength & $1,064 \mathrm{~nm}$ & {$[50]$} \\
Beam radius $w$ & $60 \mathrm{~mm}$ & {$[50]$} \\
Cavity power & $800 \mathrm{~kW}$ & {$[50]$} \\
Beamsplitter power & $2 \mathrm{~kW}$ & {$[50]$} \\
\hline
\end{tabular}

titania doped tantala and silica. Furthermore, the main contributions of thermal noise are assumed to arise from the two end mirrors and the cavity couplers. These elements are approximated to be similar regarding their thermal noise behavior. ${ }^{3}$ The mechanical, optical and thermal properties for the calculation are summarized in a previous report [49].

Figure 2 presents the results of a thermal noise analysis of an advanced detector. The optical substrates and coatings are approximated by an infinite half space. The total thermal noise of the optical components is dominated by the Brownian thermal noise of the coating. This noise is strongly dependent on the mechanical loss of the coating material itself (see Eq. (9)). While mechanical loss at room temperature easily reaches levels of below $10^{-8}$ for amorphous and crystalline bulk materials it is much higher for amorphous coating materials like tantala or silica used in multi-layer stacks. To date amorphous ion-beam-sputtered coatings are the only coatings which have been fully demonstrated to satisfy the optical requirements for the 1st and 2nd generation of gravitational wave detectors [50]. Considerable research into the dependence of the mechanical loss on different coating properties and treatments has been carried out over the last few years [22-24,36,43,52-59]. This suggests that tantala is the main source of dissipation in the silica-tantala multi-layer stacks typically used for gravitational wave detector mirror coatings, and that heat treating and doping the

\footnotetext{
3 This assumption has limitations. The cavity couplers differ from the end mirrors by the number of reflective layers and by the fact that they are transmissive optics having bulk thermo-refractive noise in addition to the other noise sources calculated. Nevertheless, the approximation used in this article is a reasonable approximation to the expected level of thermal noise.
} 
Fig. 2 Summary of total substrate thermal noise of an advanced detector. $T E$ thermo-elastic, $T R$ thermo-refractive

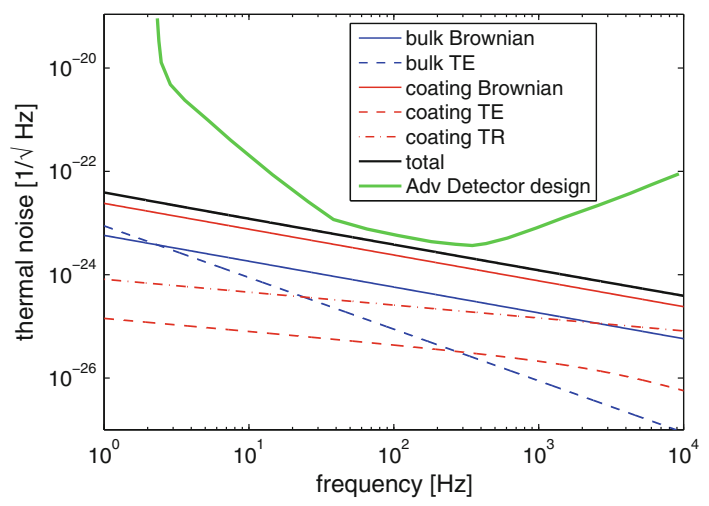

tantala component of the coatings with titania $\left(\mathrm{TiO}_{2}\right)$ can reduce the mechanical loss at room temperature by about a factor of two. Values for the mechanical loss are listed in Table 1 and were used for the calculations here. Complementary approaches to reducing mechanical loss have been proposed whereby the optical properties of a standard multi-layer stack may be achieved by using non- $\lambda / 4$ layers. These optimized coatings [60] can contain a significantly lower total amount of tantala, leading to correspondingly reduced coating thermal noise.

All other identified noise sources are well below the coating Brownian noise and are thus not expected to significantly contribute to the overall thermal noise. The total mirror thermal noise of the simplified model used here can be seen to be a close approximation to the typical design sensitivity of a 2nd generation gravitational wave detector in its most sensitive frequency range.

\section{Suspension thermal noise}

The detection principle of gravitational wave detectors is based on the measurement of the distance between 'freely falling' test masses. It is thus necessary to approximate this situation on Earth as far as can possibly be achieved-at least for displacements of the test masses along the laser beam direction. Thus, sophisticated suspensions have been developed incorporating multi-stage pendulums the final stages of which support the main detector optics. It is clear from the initial discussions on bulk materials that these suspension structures will also contribute to the overall thermal noise of the detector. Two main contributions of thermal noise originate in the suspension.

The first is pendulum thermal noise, where thermally induced motion associated with the pendulum mode of the suspension directly affects the displacement of the test mass.

The second type of thermal noise arises from the thermally induced displacements of the suspension elements under tension - their violin modes. This type of noise can cause a residual displacement of the test mass at the end of the pendulum chain. Again, thermal noise arising from these modes (on and off resonant) will contribute to the suspension thermal noise. 
There also exist other additional degrees of freedom in the suspension system which are the origin of thermal noise. One example is the so-called vertical bounce mode of the suspension. A wire under tension exhibits a vertical movement due to its internal spring constant. The (off-resonance) coupling of this motion to the main optical axes of the interferometer is small and thus will be neglected in the following treatment.

There are additional degrees of freedom in the suspension system that generate thermal noise. Each multi-stage suspension used is approximated by the last stage of the suspension chain. It shall therefore just consist of a wire of lengths $L$ under tension $M g$ with the substrate mass $M$ being considered as a point mass at the end of the wire. The upper stages of the suspension are assumed to be rigid and loss-less. The modeling of the thermal noise of a full multi-stage suspension can be found for example in $[31,61,62]$. The influence of the upper stages to the noise performance of an advanced detector was investigated in [63]. A direct approach for the suspension thermal noise is given by Braginsky et al. [64], Gonzalez and Saulson [65] and Gonzalez [66].

\subsection{Pendulum thermal noise}

A full treatment of the total thermal noise of a test mass and its pendulum suspension would again require the approach of [33], and the use of a full finite element model. This is possible but requires a reasonable amount of computational modeling effort. Instead here, the pendulum will be treated as a damped harmonic oscillator with an effective loss factor $\phi_{\text {pend }}$. Following the same treatment as described in Sect. 2 the amplitude spectral density of thermal displacement noise is then given by Saulson [31,67] and Gretarsson et al. [68]:

$$
S_{\mathrm{x}}^{\text {pend }}(f, T)=\frac{k_{\mathrm{B}} T}{2 \pi^{3} f m} \frac{f_{0}^{2} \Phi(f, T)}{\left(f^{2}-f_{0}^{2}\right)^{2}+f^{4} \Phi^{2}(f, T)} .
$$

$f$ is the frequency, $f_{0}$ the pendulum eigenfrequency. $\Phi$ is the mechanical loss describing the damping of the oscillator.

The mechanical loss $\Phi$ differs from the intrinsic loss of the suspension material as only a fraction of the total pendulum energy is stored in the elastic deformation of the suspension. The rest is stored in the gravitational field and thus is dissipation free. For $\mathrm{N}$ suspension elements the relation between intrinsic suspension material losses $\phi$ and the parameter $\Phi$ is given by Saulson [31]:

$$
\Phi=\sqrt{\frac{Y I N}{M g L^{2}}} \times \phi
$$

with the suspension material's Young's modulus $Y$ and $g$ the acceleration due to gravity. The cross-sectional moment of inertia $I$ is given for a circular fibre with diameter $d$ as:

$$
I=\frac{\pi}{64} d^{4}
$$


and for a rectangular cross-section having thickness $t$ and width $w$ :

$$
I=\frac{1}{12} w t^{3}
$$

The intrinsic suspension $\phi$ material loss consists itself of three main contributions:

$$
\phi=\phi_{\text {bulk }}+\phi_{\mathrm{TE}}+\phi_{\text {surface }}
$$

The bulk loss $\phi_{\text {bulk }}$ here is the intrinsic loss of the suspension material. Depending on the choice of the material this can be very low (e.g. $4 \times 10^{-10}$ for fused silica [51], or $5 \times 10^{-10}$ as the lowest loss measured for silicon [69]). The thermo-elastic contribution $\phi_{\mathrm{TE}}$ is given by Zener [37]:

$$
\phi_{\mathrm{TE}}=\frac{Y \alpha^{2} T}{\rho C} \times \frac{\omega \tau_{\mathrm{d}}}{1+\omega^{2} \tau_{\mathrm{d}}^{2}} .
$$

$\alpha$ is the coefficient of thermal expansion and $\tau_{\mathrm{d}}$ the thermal diffusion time given as

$$
\tau_{\mathrm{d}}=\frac{d^{2} \rho C}{13.55 \kappa}
$$

for circular fibres with a diameter of $d$ and

$$
\tau_{\mathrm{d}}=\frac{t^{2} \rho C}{\pi^{2} \kappa}
$$

for ribbons of thickness $t$.

Equation (26) does not incorporate effects arising from the tension $\sigma_{0}$ in the suspension element [70]. The surface loss $\phi_{\text {surface }}$ can be described using [68]:

$$
\phi_{\text {surface }}=\mu \alpha_{\mathrm{s}} \times \frac{S}{V}
$$

$\mu$ is a numerical factor depending on the type of vibration and the geometry. For a circular fibre under transverse motion it is 2 . In the case of a ribbon with a thickness of $t$ and a width of $w$ the factor is given by

$$
\mu=\frac{3+t / w}{1+t / w} .
$$

$\mu=3$ is a good approximation in the case of thin ribbons.

$S / V$ is the surface-to-volume ratio of the suspension and $\alpha_{S}$ a material dependent parameter describing the surface loss. $\alpha_{\mathrm{s}}$ has a dimension of a length and is typically in the range of several pm (fused silica: $3-6 \mathrm{pm}$ [71]). 


\subsection{Thermal noise from the violin modes}

The thermal noise arising from the violin modes can be determined in a similar way as the pendulum mode. Saulson calculated the thermal noise contribution from a flexible string under tension $M g$ [31]:

$$
S_{\mathrm{x}}^{\operatorname{violin}}(f, T)=\frac{k_{\mathrm{B}} T}{2 \pi^{3}} \sum_{n=1}^{\infty} \frac{\Psi_{n}^{2}(L) \phi_{n}(f, T) f_{n}^{2}}{f\left[\left(f_{n}^{2}-f^{2}\right)^{2}+\phi_{n}^{2}(f) f_{n}^{4}\right]},
$$

with

$$
\Psi_{n}^{2}(L)=\frac{\rho d^{2} L}{2 \pi M^{2}} \frac{1}{n^{2}} .
$$

Again the loss $\phi_{n}$ for each single mode consists of different contributions (bulk, thermo-elastic, surface) as described by Eq. (25). The resonant frequencies of the $n$th violin mode can be obtained from:

$$
f_{n}=\sqrt{\frac{M g}{\pi \rho d^{2} L^{2}}} \times n
$$

\subsection{Current status of the suspension thermal noise}

Fused silica is the choice not only for the test mass substrates for 2nd generation detectors but also for their suspension elements. A quasi-monolithic fused silica suspension can be formed using fused silica fibres as suspension elements [72] and using hydroxide bonding [73] to joint attachments to the sides of the test masses to which the fibres can then be welded [74]. A monolithic suspension is preferred due to the ability of avoiding excess losses from the contact points of different materials. This approach has been successfully implemented in the suspensions of the GEO600 detector $[75,76]$, and is planned for use in 2nd generation gravitational wave detectors (see, e.g. $[40,72,74,77,78])$.

Figure 3 summarizes the mechanical loss, arising from various sources, of a typical suspension element for an advanced detector. Due to the material properties of fused silica it has a thermo-elastic loss peak at around $10 \mathrm{~Hz}$ if the simple form of thermoelastic damping of the form given in Eq. (26) is considered. This loss peak occurs in a frequency region where suspension thermal noise is a significant contribution to the overall thermal noise of the detector. A detailed analysis revealed that in the case of fibres under strong tension the linear coefficient of thermal expansion should be replaced by an effective coefficient [70]:

$$
\alpha_{\mathrm{eff}} \longrightarrow \alpha-\beta \frac{\sigma_{0}}{Y}
$$

with the normalised temperature dependence of the Young's modulus $\beta=1 / Y(d Y /$ $d T$ ). If $\alpha$ and $\beta$ have the same sign (which is the case for fused silica) cancellation 
Fig. 3 Total mechanical loss of an advanced detector fused silica unloaded fibre with a diameter of $0.6 \mathrm{~mm}$
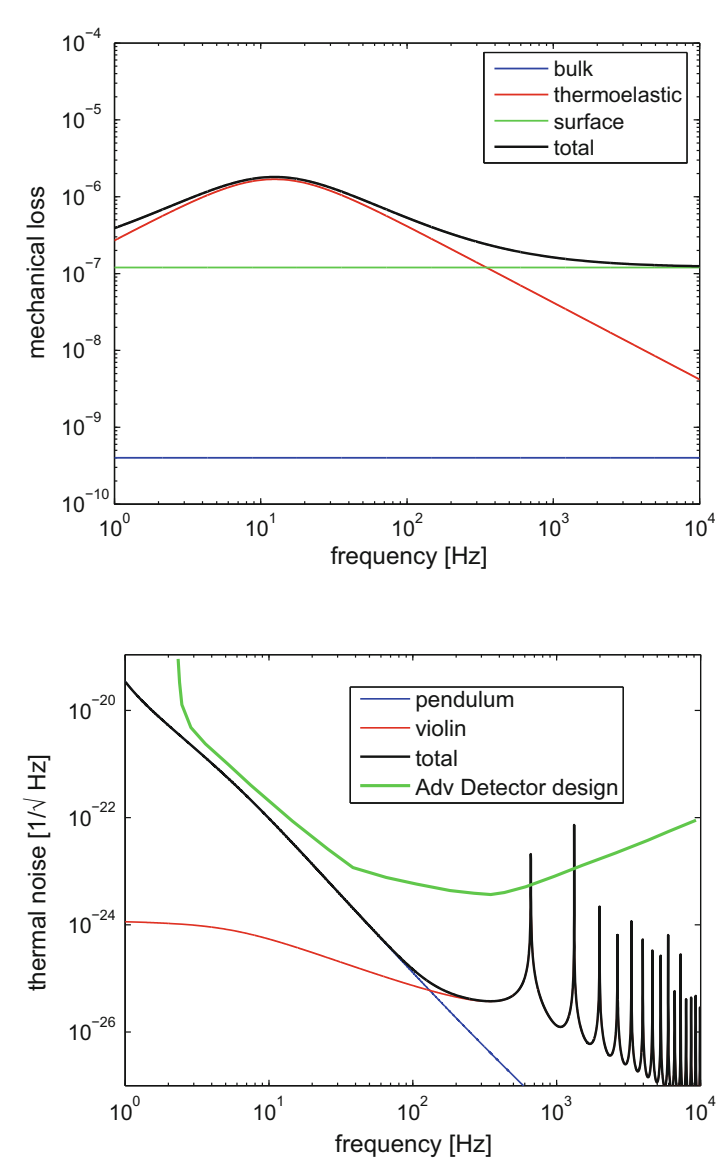

Fig. 4 Summary of the suspension thermal noise for a simplified 2rd generation detector. Parameters: $L=0.6 \mathrm{~m}$, $N=4, d=0.6 \mathrm{~mm}$. All other parameters are listed in Table 1

of the thermo-elastic contribution is possible for an appropriately tensioned fibre. For fused silica at room temperature, the necessary tension, $\sigma_{0}$, is around $200 \mathrm{MPa}$. Then the loss of the suspension element is dominated by surface loss and the thermo-elastic loss peak at low frequencies effectively vanishes (see Fig. 3).

From the discussion of surface loss in the last section, it can be seen that ribbons display a higher surface loss than fibres having the same cross sectional area [68]. Thus, when appropriately tensioned, cylindrical fibres represent an attractive choice of suspension element geometry for use in the mirror suspensions of 2nd generation detectors [40]. Additionally, this choice is supported by a better dissipation dilution and lower weld losses [74].

Combining the sources of noise discussed above allows an estimate of the overall suspension thermal noise to be made, as shown in Fig. 4. It can be seen that this is very close to the required design sensitivity of an advanced gravitational wave detector. The additional noise sources which have been neglected in this treatment (like the multi-stage pendulum, additional degrees of freedom, etc.) need to be added to the estimated curve and thus bring it closer to the design sensitivity. 


\section{Challenges in thermal noise for a 3rd generation detector}

3rd generation gravitational wave detectors should ideally have sensitivities of a factor of 10 better than that of Advanced instruments across a broad portion of the detection frequency range (see Fig. 1). As discussed earlier it is clear that advanced gravitational wave detectors will operate close to the limits of what is currently technically practical. In particular dealing with even higher stored laser powers presents a particular challenge to be overcome if sensitivities are to be further increased. In the high frequency region a gravitational wave detector is limited by photon shot noise [7]. A basic approach to reducing the effects of this noise is to increase the power in the arm cavities of the detector with estimates suggesting that powers up to $3 \mathrm{MW}$ [6] —or even higher depending on the design — might be necessary. Residual absorption in a detector optic will generate thermal variations that distort the optic. Already at the scale of 1st and 2nd generation detectors the problem of thermal lensing $[79,80]$ has been found to be significant for the stability of the interferometer. For example, the radial refraction index gradient induced when a Gaussian beam passes through an optic forms a lens which then in turn affects the laser beam propagation. Techniques exist to mitigate such effects, e.g. in 2nd generation detectors this can to some extent be compensated by the use of a suitably placed ring heater $[81,82]$ or a $\mathrm{CO}_{2}$ laser to locally heat the substrate (see, e.g. $[83,84])$.

However, it is not clear that current mitigation techniques will be scalable beyond their application in advanced detectors. Thus, the design of a 3rd generation detector is likely to need to incorporate new approaches to overcome the limitations by the currently dominating noise sources and technical limits. These include reconsideration of the optical layout of the detector, the operating temperature, the material selection for the test mass material and the coatings, and the investigation of alternative laser wavelengths if the detector needs to be operated at cryogenic temperatures [85].

\subsection{A naive approach}

It is instructive to estimate the parameters of potential 3rd generation detector based exclusively on techniques used for advanced detectors. As can be seen from the equations given earlier describing the various relevant types of thermal noise, their contribution to the overall thermal noise is a function of the laser beam radius $w$. We can ask what size of beam would be necessary to reduce the total mirror thermal noise below the desired sensitivity curve for the ET. Assuming the model of an advanced detector with the parameters shown in Table 1 the necessary beam radius $w$ (based on a Gaussian profile) to achieve the ET sensitivity presented in Fig. 1 would be $1.2 \mathrm{~m}$. Assuming the same ratios between laser beam, substrate diameter and thickness as for Advanced instruments, this would lead to a total substrate mass of 320 tons per optical component. This appears an unrealistic size even before consideration of technical issues such as the practicalities of coating or suspending the mass. Again this suggests alternative approaches rather than direct extrapolations of 2nd 
generation detector technologies are needed when going from a 2nd to a 3rd generation detector.

A significant reduction of the effects of thermal (and other) displacement noise associated with the optical components could be obtained from an extension of the interferometer arm lengths, with lengths of $10 \mathrm{~km}$ having been postulated as a goal $[5,6]$. This would reduce the mass needed from a thermal noise point of view to around 16 tons. Nevertheless, this still appears impractically high, and requires additional approaches to be implemented to reduce the mass required to a more practical level. These approaches will cover the discussion of using other materials, a different operational temperature and changes in the optical layout and the beam profile (e.g. change to higher order modes).

\subsection{Material selection process for 3rd generation detectors}

The selection of a candidate material for gravitational wave detectors is driven by a number of scientific and technical issues. Firstly, the material must have suitable optical properties (transmission, scattering, etc.). The thermal conductivity of the material then needs to be high enough to minimise the effects of the residual laser power absorbed at the surface (or in the bulk) of a mirror substrate. The material needs to be compatible with any coatings needed for the optical function of the component - as was discussed earlier both the Brownian and thermo-elastic noise of the coatings depend on substrate properties. Suitable technologies must be available for machining, polishing and coating of the material, which must be available in sufficiently large and homogeneous pieces. Recent estimates based on the calculation presented above suggest that mirror sizes of up to $60 \mathrm{~cm}$ in diameter and $40 \mathrm{~cm}$ in thickness might be needed $[6,86]$. From these points of view several materials have been considered as candidate test mass materials for future gravitational wave detectors. For operation in transmission at a laser wavelength of $1,064 \mathrm{~nm}$ (which is the wavelength of laser used in all current gravitational wave detectors) the properties of fused silica, sapphire or calcium fluoride have been investigated [87-89]. Additionally, silicon has been proposed to have excellent properties if higher wavelengths of around $1,550 \mathrm{~nm}$ are used or if a non-transmissive interferometer topology is employed $[17,79,89]$.

Silicon and sapphire for example show a 20-100 times higher thermal conductivity at room temperature than fused silica [49], making them capable of supporting higher laser powers before intolerable thermally induced mirror distortions occur. In contrast, these materials have a linear coefficient of thermal expansion which is around 10 times higher than silica and thus show a higher level of thermo-elastic noise at room temperature (see Fig. 6a), where this thermo-elastic noise then dominates the overall noise in the lower end of the operating frequency range. Thus, it is unlikely that a detector under these conditions will reach the sensitivity goal for a 3rd generation detector. However if the test mass is cooled, it's thermal properties can be very different from room temperature values, making cryogenic operation of a 3rd generation detector an option of considerable interest for study. 


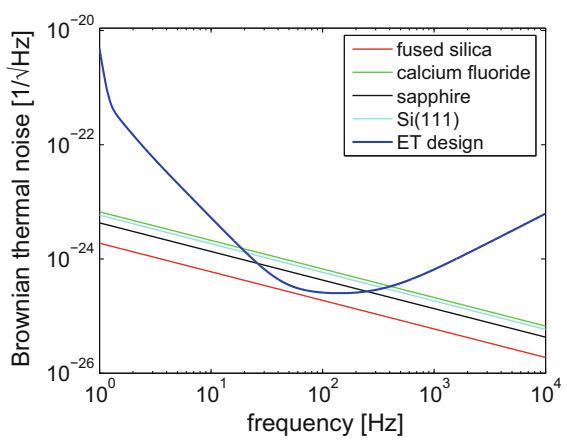

(a) $300 \mathrm{~K}$

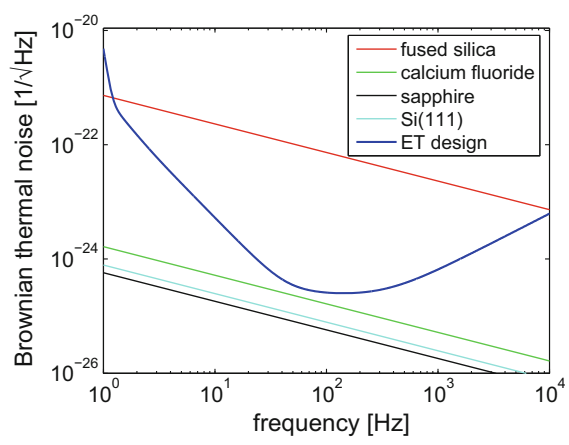

(b) $18 \mathrm{~K}$

Fig. 5 Comparison of the bulk Brownian noise for different materials. The bulk material is approximated as a semi-infinite space for simplification reasons. The beam radius was assumed to be $90 \mathrm{~mm}$ and the arm length of the detector $10 \mathrm{~km}$. $18 \mathrm{~K}$ as the cryogenic reference temperature was chosen due to the vanishing thermo-elastic contribution of silicon at this temperature (see Fig. 6)

\subsubsection{Fused silica}

Currently, all gravitational wave detectors operating at room temperature use fused silica as the optical substrate material due to a combination of excellent optical, thermal and mechanical properties. The optical absorption is lower than $0.4 \mathrm{ppm} / \mathrm{cm}$ at $1,064 \mathrm{~nm}$ [90]. The extremely low coefficient of thermal expansion at room temperature of around $5 \times 10^{-7} \mathrm{~K}^{-1}$ [91] results in a very low thermo-elastic thermal noise (see Fig. 6a). Fused silica has been shown to have a low mechanical loss at room temperature with extensive studies having been carried to on the influence of the surface quality, and type of material on the mechanical loss [51,71,76,77,92-94]. As mentioned earlier the measured mechanical loss of a sample can be considered to have components associated with both the surface and bulk of a samples with the intrinsic bulk loss potentially being as low as $4 \times 10^{-10}$ at $100 \mathrm{~Hz}$ [51].

However investigation of the acoustic absorption of amorphous silica at low temperatures [95-100] reveals an issue that makes fused silica unsuited for cryogenic use (see Fig. 5b). Studies show that a large broad mechanical loss peak exists, centered around $40 \mathrm{~K}$, associated with a double well potential associated with the $\mathrm{Si}-\mathrm{O}-\mathrm{Si}$ bonds in the amorphous structure [97,101]. This limits the mechanical loss in this region to the order of $10^{-3}$ [98].

\subsubsection{Calcium fluoride}

Calcium fluoride has been considered as a potential material for use in cryogenic gravitational wave detectors [102-105] due to its availability for commercial use in the optics industry. It is crystalline and thus should not show a broad mechanical loss peak at low temperatures like fused silica. The optical absorption of calcium fluoride is low especially in the UV range making it an ideal material for photolithography (see, e.g. [106]). 


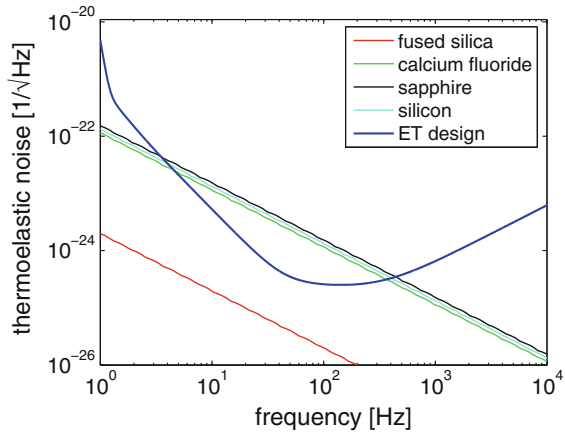

(a) $300 \mathrm{~K}$

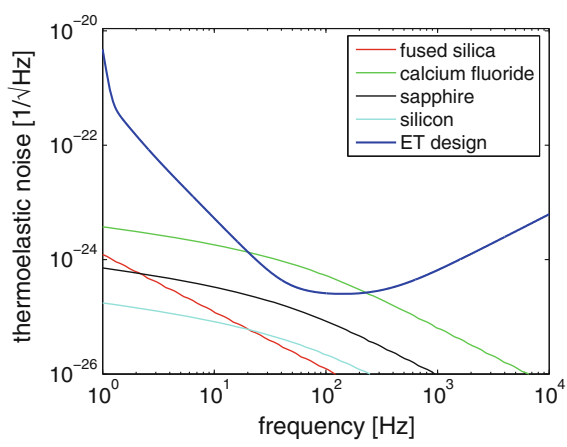

(b) $18 \mathrm{~K}$

Fig. 6 Comparison of the bulk thermo-elastic noise for different materials. The bulk material is approximated as a semi-infinite space for simplification reasons. The beam radius was assumed to be $90 \mathrm{~mm}$ and the arm length of the detector $10 \mathrm{~km}$. Near $18 \mathrm{~K}$ silicon has a linear thermal expansion coefficient of zero and therefore it is theoretically possible to cancel thermo-elastic noise. Even if the temperature is not set exactly the thermo-elastic noise contribution is very small

Studies of calcium fluoride suggest it has a higher mechanical loss [105,107,108] than sapphire or silicon. Furthermore, the high thermal expansion coefficient results in a relatively high level of thermo-elastic noise (see Fig. 6) and makes the material very sensitive to thermal shocks.

\subsubsection{Sapphire}

Sapphire has a hexagonal crystal structure making it anisotropic in several mechanical, thermal and optical properties. Sapphire can have excellent transmission at $1064 \mathrm{~nm}$ and a low $d n / d T$ [49]. It's high Young's modulus of about $400 \mathrm{GPa}$ [28] and high thermal conductivity of up to $40 \mathrm{~W} / \mathrm{m} \times \mathrm{K}$ [28] make it a promising candidate material for a substrate material capable of tolerating exposure to high laser powers. Use at room temperature is limited due to it's thermal expansion coefficient of $5.1 \times 10^{-6} \mathrm{~K}^{-1}$ [28] and thus a higher thermo-elastic noise level than would be obtained with, e.g. fused silica (see Fig. 6). Properties of sapphire as a cryogenic substrate material have been studied by several groups [87,109-111] suggesting that sapphire has a very low mechanical loss. At cryogenic temperatures it reaches $2 \times 10^{-10}$ [87]. Furthermore, the use of suspension elements made from monocrystalline sapphire fibres has been studied [112,113].

\subsubsection{Silicon}

Silicon presents another interesting candidate substrate material for cryogenic use $[17,79]$. At room temperature silicon has a relatively large thermal expansion coefficient and thus shows a high level of thermo-elastic noise compared to fused silica (see Fig. 6). The temperature dependence of the linear thermal expansion coefficient exhibits two temperatures (around 18 and $125 \mathrm{~K}$ ) where this coefficient is zero [114]. This makes silicon interesting because thermo-elastic effects will be nulled (see Eq. (5) 
and (26)). In addition the cancellation of thermo-elastic effects in suspension elements (see Eq. (34)) under tension is a possibility here. In silicon the temperature dependence $\beta$ of Young's modulus has a negative sign as temperature is reduced below room temperature. Thus, only in temperature regions where $\alpha$ is also negative (between 18 and $125 \mathrm{~K}$ for silicon) is it possible to cancel the thermo-elastic noise of suspension elements by loading them with an appropriate tension.

Silicon has a high thermal conductivity at low temperatures showing a peak at around $20 \mathrm{~K}$ [114]. Young's modulus is significantly higher than for fused silica and dependents on the crystal orientation. The [111] direction shows the highest Young's modulus of $188 \mathrm{GPa}$ [115] among all other orientations.

The mechanical loss of silicon bulk samples was studied in the late 1970s [69] with very low mechanical loss found at temperatures below $10 \mathrm{~K}$. At around $20 \mathrm{~K}$ a loss peak was observed which was postulated to be associated with sample impurities (oxygen, doping concentration, etc.). In later experiments this mechanical loss peak was not observed due possibly to an improvement in the silicon purification, however the range of factors influencing the temperature dependent mechanical loss of silicon are not wholly understood and studies of this are ongoing (see, e.g. [116-118]), including the effects of doping.

Silicon is available in relatively large pieces, up to $500 \mathrm{~kg}$ driven by the requirements of the semiconductor industry. Additionally, sophisticated silicon (micro) machining and polishing technology exists, makes silicon interesting for the application novel approaches like the use of gratings or other all-reflective elements as described briefly in Sect. 5. A variety of techniques exist for bonding silicon, e.g. direct bonding [119] or silicate bonding of oxidized samples [120] allowing for the possibility of constructing quasi-monolithic silicon suspensions.

Nevertheless, if silicon is used for transmissive optics (beam splitters, cavity couplers) the wavelength of laser used to illuminate such optics needs to be shifted towards larger wavelengths due to the absorptive properties of silicon. Nd:YAG laser light at $1,064 \mathrm{~nm}$ is close to the fundamental absorption of silicon due to its band gap of $1.1 \mathrm{eV}$ [114]. A promising laser technology based on Er:YAG or Er-fibre lasers at around $1,550 \mathrm{~nm}$ is under strong development [121] and could enable low optical absorption $<1 \mathrm{ppm} / \mathrm{cm}$ [121]. Furthermore, even if all-reflective topologies are used a shift to a higher operating wavelength could have advantages. Each optical layer has a reflectivity of $<100 \%$. The remaining laser light is scattered or transmitted. The transmitted part will be absorbed by the substrate if this is not transparent. Using $1,550 \mathrm{~nm}$ would mean that even the small amount of transmitted light from the optical coatings would be transmitted and thus would not cause heating in the elements. If a stored beam power of up to $3 \mathrm{MW}$ within the cavity is used this will be a significant improvement and ease the design of cooling techniques needed [62].

On the other hand if the wavelength used is increased the thickness of the layers required in optical coating layers must also be increased. This leads to increased Brownian coating noise. Thus, very low noise coatings are needed in order to avoid negating the advantages of silicon at $1,550 \mathrm{~nm}$. Alternative approaches to creating highly reflective surfaces by using monolithic optics based on microstructured surfaces are currently under development [122] and investigation (see Sect. 5). 


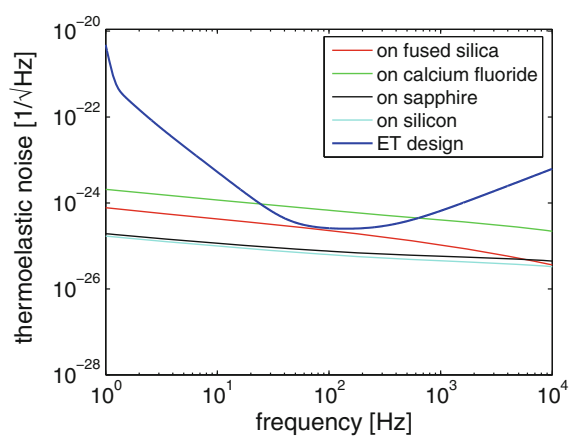

(a) $300 \mathrm{~K}$

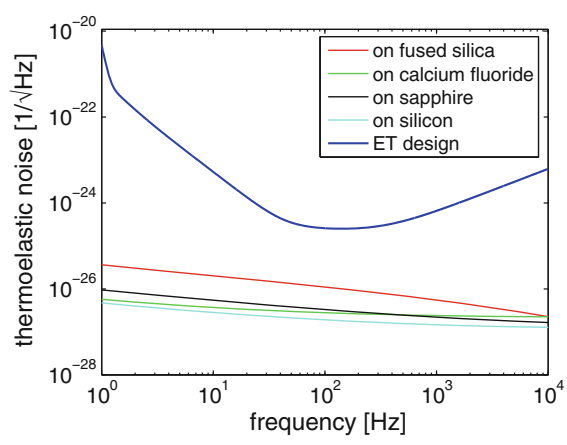

(b) $18 \mathrm{~K}$

Fig. 7 Comparison of the contribution of the coating thermo-elastic noise of a tantala/silica multilayer (18 doublets) on different materials. The beam radius was assumed to be $90 \mathrm{~mm}$ and the arm length of the detector $10 \mathrm{~km}$

\subsubsection{Coating materials}

As discussed earlier coating thermal noise is expected to dominate the total thermal noise of the main optical components in gravitational wave detectors (see Sect. 2.3). Thus, it will be one of the most critical issues in designing 3rd generation optics. Investigations of typical silica-tantala coating properties at cryogenic temperatures have been carried out $[58,59]$ revealing a loss peak at around $18 \mathrm{~K}$ for tantala. This loss peak may in part negate the gains in reducing coating Brownian noise by going to cryogenic temperatures, however an improvement of about a factor of two is still found when the effects of the increased mechanical loss towards $18 \mathrm{~K}$ are included. Results from the Japanese groups obtained from multilayer coatings have not shown these loss peaks at cryogenic temperatures at all [53]. Currently, several groups are investigating the temperature dependence of the mechanical loss in order to understand these results. So far the heat treatment needed in order to achieve the optical requirements seems to have an influence on the mechanical loss and might be the reason why loss peaks occur. Detailed experimental investigations are underway.

Figure 7 shows the level of coating thermo-elastic noise expected for different multi-layer-bulk material combinations. The multilayer stack is assumed to be an 18 doublet stack of $\lambda / 4$ layers of tantala and silica at $1,064 \mathrm{~nm}$. The lowest contribution at room temperature as well as at cryogenic temperatures arises from the combination multi-layer-silicon. Silicon is compatible with the current coating techniques and thus would be a suitable bulk material from the coating point of view.

While tantala and silica have been studied extensively at low temperatures other coating materials have not been investigated in such detail to date. A summary of the thermal, mechanical and optical properties of current coating materials can be found in [49]. Nevertheless, all other coating materials showed a higher mechanical loss making them less attractive for a 3rd generation detector. 


\subsection{Thermal noise of a 3rd generation detector based on current technologies}

In order to estimate the thermal noise of a 3rd generation detector, a simplified model similar to that used for 2nd generation detectors is used here as a first approach (see Sects. 2.3 and 3.3). The main thermal noise contributions are again assumed to arise from the end mirrors and the cavity couplers. An arm length of $10 \mathrm{~km}$ and a circulating laser power of $3 \mathrm{MW}$ is used [6]. The optical components are assumed to be identical for simplicity having 18 doublets of $\lambda / 4$ layers of titania doped tantala and silica.

We will focus from that stage onwards on the optimisation of a single detector operating at cryogenic temperatures as a first approach for a 3rd generation detector. However, this is clearly not the only possible approach. It shall rather be used to demonstrate the capabilities and limits of this approach.

First the thermal noise of the mirror was estimated. $\mathrm{Si}(111)$ is chosen as the test mass material due to its high Young's modulus. In order to reduce the different thermal noise contributions an increase in laser beam radius is desirable, requiring an increase in substrate diameter in order to keep beam clipping losses as small as possible. For Advanced LIGO a substrate-to-beam diameter ratio of 2.8 was chosen (see Table 1). If the laser beam radius $w$ is increased moderately from $60 \mathrm{~mm}$ up to say $90 \mathrm{~mm}$ for a 3 rd generation detector this would lead to a substrate diameter of $510 \mathrm{~mm}$. This seems to be a not unreasonable size assuming continuing commercial development of the silicon single crystal market. The semiconductor industry is currently investigating the possibility of using $20 \mathrm{in}$. $(508 \mathrm{~mm})$ diameter wafers thus making silicon available of this diameter when a 3rd generation detector might be built. Of course, a further increase of the laser beam diameter would further reduce thermal noise but it seems unlikely currently that samples with the required purity would be available in the near future. Thus, the following estimates are based on a $90 \mathrm{~mm}$ beam radius and a $510 \mathrm{~mm}$ diameter silicon substrate. Keeping the same aspect ratio as for Advanced LIGO would lead to a substrate thickness of about $300 \mathrm{~mm}$.

With the use of high laser powers comes noise due to radiation pressure, as photons are reflected from the interferometer mirrors (see, e.g. [67]). To keep this noise below the levels desired for ET a minimum mass was estimated by Hild et al. [6] to be around $120-150 \mathrm{~kg}$. For a $510 \mathrm{~mm}$ diameter substrate this coincides with the thickness of about $300 \mathrm{~mm}$ which was determined above for a constant aspect ratio. This test mass geometry seems to be plausible and is realistic to assume being available within the next years.

Figure 8 shows the noise contributions discussed in Sect. 2 based on literature values for the mechanical and thermal properties of silicon. A detailed description of this calculation can be found in $[49,86]$.

At $300 \mathrm{~K}$ the detector built with silicon substrates would not reach the required ET design sensitivity. The total noise is almost totally dominated by the thermo-elastic noise of the bulk material itself. At $18 \mathrm{~K}$ the thermo-elastic noise of the silicon bulk material disappears due to the vanishing coefficient of thermal expansion. The total noise is reduced significantly due to the reduced temperature but mainly due to a large change in material properties (see [49]). The total noise is below the desired ET sensitivity curve apart from frequencies between 40 and $80 \mathrm{~Hz}$. Here, the total thermal noise is limited due to coating Brownian noise caused by the increasing mechanical 


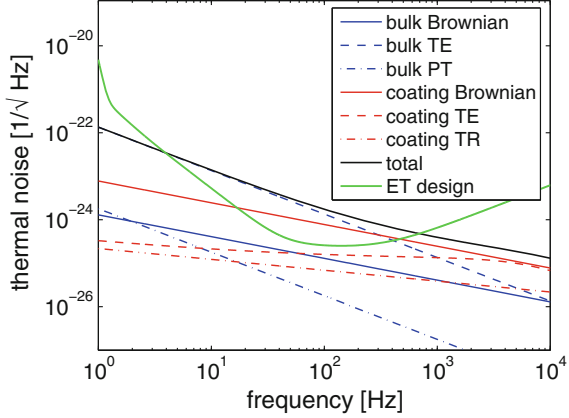

(a) $300 \mathrm{~K}$

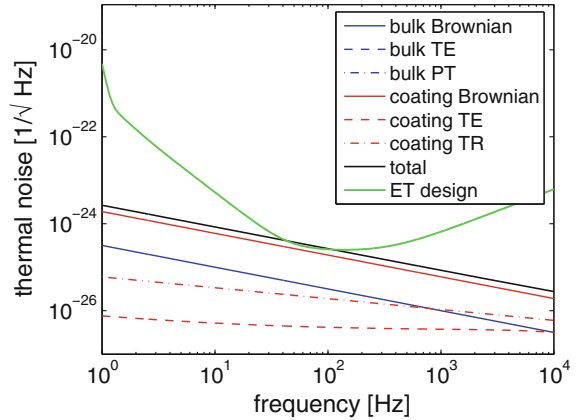

(b) $18 \mathrm{~K}$

Fig. 8 Thermal noise contribution for a potential 3rd generation mirror made of $\operatorname{Si}(111)$. Beam radius $w=90 \mathrm{~mm}$ arm length $10 \mathrm{~km}$. $T E$ thermo-elastic, $T R$ thermo-refractive

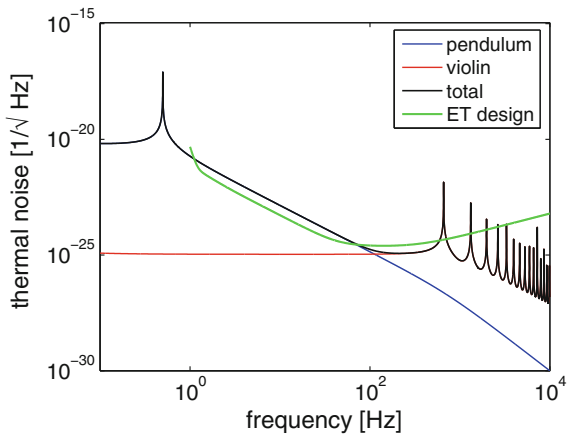

(a) $300 \mathrm{~K}$

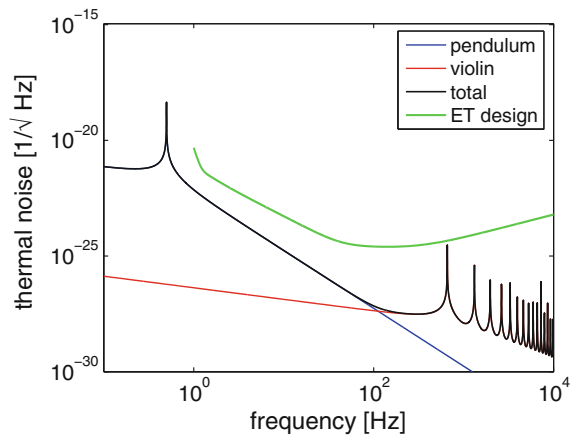

(b) $18 \mathrm{~K}$

Fig. 9 Suspension thermal noise for four $680 \mu \mathrm{m} \mathrm{Si(100)} \mathrm{fibres} \mathrm{and} \mathrm{a} \mathrm{substrate} \mathrm{mass} \mathrm{of} 150 \mathrm{~kg}$

loss of the coating materials at cryogenic temperatures. Around $18 \mathrm{~K}$ the mechanical loss of tantala is limited to about $8 \times 10^{-4}[58,59]$. This reveals that for a realistic design of a 3rd generation detector based on our current technological and scientific knowledge the coatings need to be further improved. A factor of 2 in reduction of the mechanical loss at cryogenic temperatures of silica or tantala would be sufficient to reduce the total thermal noise below the ET sensitivity curve.

The next step is the calculation of suspension thermal noise. Research in this area is still in its initial phase and thus only a simple estimate using a single-stage pendulum is given. $\mathrm{Si}(100)$ was assumed to be the suspension material. The $\mathrm{Si}(100)$ orientation shows the lowest possible Young's modulus among all other orientations [115] and thus the lowest suspension thermal noise (see Eqs. (22) and (26)). The pendulum length of the last stage is chosen to be $1 \mathrm{~m}$ as a starting point based on plausible fabrication techniques [123]. Silicon shows a breaking strength of about $0.2-7 \mathrm{GPa}$ [124]. Assuming a tension per fibre of about $1 \mathrm{GPa}$ would result in a fibre diameter of $680 \mu \mathrm{m}$ assuming four identical suspension elements (see. Fig. 9). 
Fig. 10 Estimated total thermal noise of a potential $3 \mathrm{rd}$ generation detector

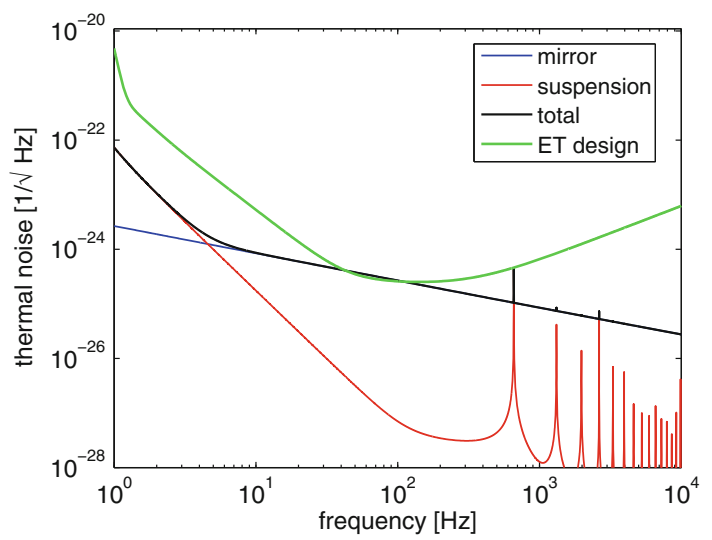

At room temperature the suspension element is dominated by thermo-elastic noise due to the large linear thermal expansion coefficient. As mentioned earlier compensation of suspension thermo-elastic noise at room temperature is not possible in silicon due to the opposite signs of $\alpha$ and $\beta .^{4}$ The level of noise exceeds the limits for ET. However cooling to about $18 \mathrm{~K}$ removes the thermo-elastic contribution to the overall mechanical loss of the fibre and thus results in a suspension thermal noise which is lower than the goal for a 3rd generation detector.

Figure 10 summarizes the total thermal noise of the simplified 3rd generation detector. The current model does not yet include additional possible noise sources like any losses associated with jointing the suspension elements to the test masses. Furthermore, the suspension system considered is a very simplified model, nevertheless, the noise estimates obtained are encouraging.

\section{Possible techniques to further reduce thermal noise}

So far our estimates suggest that the desired sensitivity for ET may be reached from a thermal noise point of view. However since our model of the test masses and the suspension is still not complete enough to account for all noise sources it is preferable to start with a concept that further reduce the effect of thermal noise on the sensitivity of the detector, allowing more flexibility during the design phase of such a detector.

Given the high laser powers considered it would be desirable to avoid transmissive optics completely if possible. During recent years there have been proposals for interferometers based exclusively on reflective components $[10,13]$. Beam splitters and cavity couplers can be replaced by sophisticated grating optics allowing good optical performance [12]. This may possibly lead to components having lower optical absorption and no thermal lensing. Nevertheless, these components still rely on the use of reflective dielectric multilayer stacks having some residual absorption and will suffer from coating thermal noise. Furthermore, it has been shown that grating optics of this

\footnotetext{
4 The sign of the parameter $\beta$ can be obtained from the temperature dependence of the resonant frequency of silicon oscillators.
} 
type need stabilization of their lateral motion in order not to introduce additional phase noise [125].

Another promising approach is to investigate the dependence of the read-out noise on the shape of the laser beam. So far, all results presented in this article rely on the use of Gaussian laser beam having a beam radius of $w$. Changing to other beam shapes like Mesa beams [126] or higher order Laguerre-Gaussian modes [14] may be possible. This leads to a more homogeneous illumination of the mirror surface and thus a better averaging of thermal noise. The use of these exotic beams in gravitational wave detectors is still under development, with the propagation of these beams and the impact on thermal noise being investigated $[60,127]$. The generation of these types of laser modes with high laser powers is a technical issue which also needs to be investigated [128-131].

A third technique tackles the main thermal noise source-the Brownian coating noise. Several possibilities have been proposed to reduce the thickness of the dielectric coating material [60]. This would immediately impact Brownian thermal noise (see Eq. (9)). Another recent proposal is to use resonant waveguide mirrors. Here, reflection is produced by coupling the light to a waveguide by means of a grating structure [132]. If designed properly high reflectivity can be achieved using only one dielectric layer with a thickness of about $400 \mathrm{~nm}$. Compared to the total thickness of several $\mu \mathrm{m}$ of a current multilayer stack this should lead to a decreased level of coating Brownian noise.

In a recent step the idea of a resonant waveguide led to a monolithic waveguide structure made purely from silicon [122]. The idea is to form the resonant waveguide (which needs to have a lower refraction index than the adjoining layers for propagation purposes) by means of an effective media. This mirror may have a very low mechanical loss and thus small thermal noise. However due to the etched surfaces needed for the effective medium it is very likely to have an increased level of surface loss for these components, and studies of this are underway. An additional advantage of these silicon structures could be a decreased level of optical absorption if the longer wavelengths whose usage was discussed above are employed. Up to now these resonant waveguide mirrors (both dielectric and monolithic) have been developed to potentially replace cavity coupler or end mirrors, however the construction of a beam splitter based on these novel techniques has not yet been achieved and is a subject requiring further study.

We note that all optical components based on gratings need to be fabricated with very high accuracy on the large surface areas needed for a 3rd generation detector, since although the reduced level of thermal noise expected from use of these gratings would allow a reduction in the diameter of the test mass it is unlikely to reduce the diameter below $25 \mathrm{~cm}$. Up to now the gratings of this type have been fabricated on thin wafers and produce these micro- and nanostructures on $150 \mathrm{~kg}$ test masses directly seems currently impractical. Thus, a technique to allow thin microstructured substrates to be bonded to large size test masses would be required, and any mechanical losses introduced by this bonding would need to be investigated. Additional investigations of light scattering of these structures is needed. Light scattering is already a serious issue in 1st and 2nd generation detectors and will clearly be important for 3rd generation detectors as well. 


\section{Conclusions}

We have summarised the current state of the art of thermal noise treatment in gravitational wave detectors. Starting from a baseline of the materials and techniques used for 2nd generation detectors we discussed several possible options for further improving thermal noise. The aim of an increased over all sensitivity by a factor of 10 by going towards the 3rd generation detectors is a challenging scientific and engineering work. Thermal noise will be a serious obstacle because the technology used for 2 nd generation detectors seems to have reached its limit. Thus, a change in the material and operational regime needs to be done. We have summarised the selection of potential bulk, coating and suspension materials. In general, the use of silicon as an optical material at cryogenic temperatures around $20 \mathrm{~K}$ seems to be promising due to the excellent properties and the foreseeable availability in large enough ingots. Further $\mathrm{R} \& \mathrm{D}$ is needed in order to obtain the desired total performance of a 3rd generation detectors. For example, the techniques for a further reduction of coating Brownian noise or the fabrication of monolithic suspension elements made of silicon need to be investigated and will stimulate the research within this field for the next several years.

In this paper we have presented strong evidence that, with focused R\&D over the next several years, the estimated thermal noise for a 3rd generation detector should be able to meet the design sensitivity requirements.

Acknowledgments We would like to thank our colleagues in the GEO600 project, the LSC-Virgo collaboration, the ET project and within SUPA for their interest in this work. We thank Y. Levin for very helpful comments on this paper. We are grateful for the financial support provided by Science and Technology Facilities Council (STFC), the Leverhulme Trust, the Scottish Funding Council (SFC) and the University of Glasgow in the UK. This work was in part supported by the European Union under a FP7 project (Grant Agreement 211743). This paper has LIGO DCC number LIGO-P0900123.

\section{References}

1. Flaminio, R., Freise, A., Gennai, A., Hello, P., Penna, P.L., Lusordo, G., Lueck, H., Man, N., Masserot, A., Mours, B., Punturo, M., Spallicci, A., Vicere, A.: Advanced Virgo white paper. Virgo note VIR-NOT-DIR-1390-304 (2005). http://wwwcascina.virgo.infn.it/advirgo/docs/whitepaper.pdf

2. Weinstein, A.: Class. Quantum Grav. 19(7), 1575 (2002)

3. Abbott, R., Adhikari, R., Ballmer, S., Barsotti, L., Evans, M., Fritschel, P., Frolov, V., Mueller, G., Slagmolen, B., Waldman, S.: AdvLIGO interferometer sensing and control conceptual design. Ligo Document Center, LIGO-T070247-01-1 (2007). http://dcc.ligo.org/

4. Whitcomb, S.E.: Class. Quantum Grav. 25(11), 114013 (2008)

5. Punturo, M., Lueck, H.: FP7 Design study proposal (2007). http://www.ego-gw.it/ILIAS-GW/ FP7-DS/fp7-DS.htm

6. Hild, S., Chelkowski, S., Freise, A.: Pushing towards th ET sensitivity using 'conventional' technology. ET technical note ET-010-08 (2008). arXiv:0810.0604 v2 [gr-qc]

7. Caves, C.M.: Phys. Rev. D 23(8), 1693 (1981)

8. McKenzie, K., Grosse, N., Bowen, W.P., Whitcomb, S.E., Gray, M.B., McClelland, D.E., Lam, P.K.: Phys. Rev. Lett. 93(16), 161105 (2004)

9. Vahlbruch, H., Mehmet, M., Chelkowski, S., Hage, B., Franzen, A., Lastzka, N., ler, S.G., Danzmann, K., Schnabel, R.: Phys. Rev. Lett. 100(3), 033602 (2008)

10. Sun, K.X., Byer, R.L.: Opt. Lett. 23(8), 567 (1998)

11. Wise, S., Quetschke, V., Deshpande, A.J., Mueller, G., Reitze, D.H., Tanner, D.B., Whiting, B.F., Chen, Y., Tünnermann, A., Kley, E.B., Clausnitzer, T.: Phys. Rev. Lett. 95(1), 013901 (2005) 
12. Bunkowski, A., Burmeister, O., Clausnitzer, T., Kley, E.B., Tünnermann, A., Danzmann, K., Schnabel, R.: Appl. Opt. 45(23), 5795 (2006)

13. Fahr, S., Clausnitzer, T., Kley, E.B., Tünnermann, A.: Appl. Opt. 46(24), 6092 (2007)

14. Vinet, J.Y.: Class. Quantum Grav. 22(7), 1395 (2005)

15. Bondarescu, M., Thorne, K.S.: Phys. Rev. D 74(8), 082003 (2006)

16. Lovelace, G.: Class. Quantum Grav. 24(17), 4491 (2007)

17. Rowan, S., Hough, J., Crooks, D.: Phys. Lett. A 347(1-3), 25 (2005)

18. Saulson, P.R.: Phys. Rev. D 30(4), 732 (1984)

19. Hughes, S.A., Thorne, K.S.: Phys. Rev. D 58(12), 122002 (1998)

20. Shoemaker, D.: Class. Quantum Grav. 20(10), S11 (2003)

21. Conforto, G., DeSalvo, R.: Nucl. Instrum. Methods Phys. Res. A 518(1-2), 228 (2004)

22. Harry, G.M., Gretarsson, A.M., Saulson, P.R., Kittelberger, S.E., Penn, S.D., Startin, W.J., Rowan, S., Fejer, M.M., Crooks, D.R.M., Cagnoli, G., Hough, J., Nakagawa, N.: Class. Quantum Grav. 19(5), 897 (2002)

23. Crooks, D.R.M., Sneddon, P., Cagnoli, G., Hough, J., Rowan, S., Fejer, M.M., Gustafson, E., Route, R., Nakagawa, N., Coyne, D., Harry, G.M., Gretarsson, A.M.: Class. Quantum Grav. 19(5), 883 (2002)

24. Harry, G.M., Armandula, H., Black, E., Crooks, D.R.M., Cagnoli, G., Hough, J., Murray, P., Reid, S., Rowan, S., Sneddon, P., Fejer, M.M., Route, R., Penn, S.D.: Appl. Opt. 45(7), 1569 (2006)

25. Einstein, A.: Ann. Phys. 17, 549 (1905)

26. Nowick, A.S., Berry, B.S.: Anelastic Relaxation in Crystalline Solids. Academic Press, London (1972)

27. Braginsky, V.B., Gorodetsky, M.L., Vyatchanin, S.P.: Phys. Lett. A 264(1), 1 (1999)

28. Braginsky, V.B., Gorodetsky, M.L., Vyatchanin, S.P.: Phys. Lett. A 271(5-6), 303 (2000)

29. Callen, H.B., Welton, T.A.: Phys. Rev. 83(1), 34 (1951)

30. Callen, H.B., Greene, R.F.: Phys. Rev. 86(5), 702 (1952)

31. Saulson, P.R.: Phys. Rev. D 42(8), 2437 (1990)

32. Gillespie, A., Raab, F.: Phys. Rev. D 52(2), 577 (1995)

33. Levin, Y.: Phys. Rev. D 57(2), 659 (1998)

34. Bondu, F., Hello, P., Vinet, J.Y.: Phys. Lett. A 246, 227 (1998)

35. Liu, Y.T., Thorne, K.S.: Phys. Rev. D 62(12), 122002 (2000)

36. Yamamoto, K., Ando, M., Kawabe, K., Tsubono, K.: Phys. Lett. A 305(1-2), 18 (2002)

37. Zener, C.: Phys. Rev. 53(1), 90 (1938)

38. Rowan, S., Twyford, S., Hutchins, R., Hough, J.: Class. Quantum Grav. 14(6), 1537 (1997)

39. Rowan, S., Twyford, S.M., Hutchins, R., Kovalik, J., Logan, J.E., McLaren, A.C., Robertson, N.A., Hough, J.: Phys. Lett. A 233(4-6), 303 (1997)

40. Cumming, A., Heptonstall, A., Kumar, R., Cunningham, W., Torrie, C., Barton, M., Strain, K., Hough, J., Rowan, S.: Class. Quantum Grav. 26(21), 215012 (2009)

41. Cerdonio, M., Conti, L., Heidmann, A., Pinard, M.: Phys. Rev. D 63(8), 082003 (2001)

42. Nakagawa, N., Gretarsson, A.M., Gustafson, E.K., Fejer, M.M.: Phys. Rev. D 65(10), 102001 (2002)

43. Penn, S.D., Sneddon, P.H., Armandula, H., Betzwieser, J.C., Cagnoli, G., Camp, J., Crooks, D.R.M., Fejer, M.M., Gretarsson, A.M., Harry, G.M., Hough, J., Kittelberger, S.E., Mortonson, M.J., Route, R., Rowan, S., Vassiliou, C.C.: Class. Quantum Grav. 20(13), 2917 (2003)

44. Somiya, K., Yamamoto, K.: Phys. Rev. D 79(10), 102004 (2009)

45. Braginsky, V.B., Vyatchanin, S.P.: Phys. Lett. A 312(3-4), 244 (2003)

46. Fejer, M.M., Rowan, S., Cagnoli, G., Crooks, D.R.M., Gretarsson, A., Harry, G.M., Hough, J., Penn, S.D., Sneddon, P.H., Vyatchanin, S.P.: Phys. Rev. D 70(8), 082003 (2004)

47. Levin, Y.: Phys. Lett. A 372(12), 1941 (2008)

48. Evans, M., Ballmer, S., Fejer, M., Fritschel, P., Harry, G., Ogin, G.: Phys. Rev. D 78(10), 102003 (2008)

49. Franc, J., Morgado, N., Flaminio, R., Nawrodt, R., Martin, I., Cunningham, L., Cumming, A., Rowan, S., Hough. J.: Mirror thermal noise in laser interferometer gravitational wave detectors operating at room and cryogenic temperature. ET document ET-021-09 (2009). http://www.et-gw.eu/

50. Advanced LIGO Team. Advanced LIGO Reference Design: Ligo Document Center, LIGO-06005608-M (2007). http://dcc.ligo.org/ 
51. Penn, S.D., Ageev, A., Busby, D., Harry, G.M., Gretarsson, A.M., Numata, K., Willems, P.: Phys. Lett. A 352(1-2), 3 (2006)

52. Harry, G.M., Abernathy, M.R., Becerra-Toledo, A.E., Armandula, H., Black, E., Dooley, K., Eichenfield, M., Nwabugwu, C., Villar, A., Crooks, D.R.M., Cagnoli, G., Hough, J., How, C.R., MacLaren, I., Murray, P., Reid, S., Rowan, S., Sneddon, P.H., Fejer, M.M., Route, R., Penn, S.D., Ganau, P., Mackowski, J.M., Michel, C., Pinard, L., Remillieux, A.: Class. Quantum Grav. 24(2), 405 (2007)

53. Yamamoto, K., Miyoki, S., Uchiyama, T., Ishitsuka, H., Ohashi, M., Kuroda, K., Tomaru, T., Sato, N., Suzuki, T., Haruyama, T., Yamamoto, A., Shintomi, T., Numata, K., Waseda, K., Ito, K., Watanabe, K.: Phys. Rev. D 74(2), 022002 (2006)

54. Black, E.D., Villar, A., Barbary, K., Bushmaker, A., Heefner, J., Kawamura, S., Kawazoe, F., Matone, L., Meidt, S., Rao, S.R., Schulz, K., Zhang, M., Libbrecht, K.G.: Phys. Lett. A 328(1), 1 (2004)

55. Crooks, D.R.M., Cagnoli, G., Fejer, M.M., Harry, G., Hough, J., Khuri-Yakub, B.T., Penn, S., Route, R., Rowan, S., Sneddon, P.H., Wygant, I.O., Yaralioglu, G.G.: Class. Quantum Grav. 23(15), 4953 (2006)

56. Amico, P., Bosi, L., Cottone, F., Dari, A., Gammaitoni, L., Marchesoni, F., Punturo, M., Travasso, F., Vocca, H.: J. Phys. Conf. Ser. 32(1), 413 (2006)

57. Abernathy, M.R., Harry, G.M., Travasso, F., Martin, I., Reid, S., Rowan, S., Hough, J., Fejer, M.M., Route, R., Penn, S., Armandula, H., Gretarsson, A.: Phys. Lett. A 372(2), 87 (2008)

58. Martin, I., Armandula, H., Comtet, C., Fejer, M.M., Gretarsson, A., Harry, G., Hough, J., Mackowski, J.M.M., MacLaren, I., Michel, C., Montorio, J.L., Morgado, N., Nawrodt, R., Penn, S., Reid, S., Remillieux, A., Route, R., Rowan, S., Schwarz, C., Seidel, P., Vodel, W., Zimmer, A.: Class. Quantum Grav. 25(5), 055005 (2008)

59. Martin, I.W., Chalkley, E., Nawrodt, R., Armandula, H., Bassiri, R., Comtet, C., Fejer, M.M., Gretarsson, A., Harry, G., Heinert, D., Hough, J., MacLaren, I., Michel, C., Montorio, J.L., Morgado, N., Penn, S., Reid, S., Route, R., Rowan, S., Schwarz, C., Seidel, P., Vodel, W., Woodcraft, A.L.: Class. Quantum Grav. 26(15), 155012 (2009)

60. Agresti, J., Castaldi, G., DeSalvo, R., Galdi, V., Pierro, V., Pinto, I.M.: Optimized multilayer dielectric mirror coatings for gravitational wave interferometers. LIGO Document Center, LIGO-P060027-00-Z (2006). http://dcc.ligo.org/

61. Majorana, E., Ogawa, Y.: Phys. Lett. A 233(3), 162 (1997)

62. Puppo, P.: A thermal noise model for a branched system of mechanical harmonic oscillators. Poster presented at the 8th Edoardo Amaldi Conference in New York (2009). http://www.amaldi8.org/

63. Piergiovanni, F., Punturo, M., Puppo, P.: The thermal noise of the Virgo+ and Virgo Advanced Last Stage Suspension. Virgo note VIR-0015C-09 (2009). https://tds.ego-gw.it/ql/?c=2199

64. Braginsky, V.B., Levin, Y., Vyatchanin, S.P.: Meas. Sci. Technol. 10(7), 598 (1999)

65. Gonzalez, G.I., Saulson, P.R.: J. Acoust. Soc. Am. 96(1), 207 (1994)

66. Gonzalez, G.: Class. Quantum Grav. 17(21), 4409 (2000)

67. Saulson, P.R.: Fundamentals of Interferometric Gravitational Wave Detectors. World Scientific Publishing Co Pte Ltd, Singapore (1994)

68. Gretarsson, A.M., Harry, G.M., Penn, S.D., Saulson, P.R., Startin, W.J., Rowan, S., Cagnoli, G., Hough, J.: Phys. Lett. A 270(3-4), 108 (2000)

69. McGuigan, D.F., Lam, C.C., Gram, R.Q., Hoffman, A.W., Douglas, D.H., Gutche, H.W.: J. Low Temp. Phys. 30, 621 (1978)

70. Cagnoli, G., Willems, P.A.: Phys. Rev. B 65(17), 174111 (2002)

71. Gretarsson, A.M., Harry, G.M.: Rev. Sci. Instrum. 70(10), 4081 (1999)

72. Cagnoli, G., Armandula, H., Cantley, C.A., Crooks, D.R.M., Cumming, A., Elliffe, E., Fejer, M.M., Gretarsson, A.M., Harry, G.M., Heptonstall, A., Hough, J., Jones, R., Mackowski, J.M., Martin, I., Murray, P., Penn, S.D., Perreur-Lloyd, M., Reid, S., Route, R., Rowan, S., Robertson, N.A., Sneddon, P.H., Strain, K.A.: J. Phys. Conf. Ser. 32(1), 386 (2006)

73. Rowan, S., Twyford, S.M., Hough, J., Gwo, D.H., Route, R.: Phys. Lett. A 246(6), 471 (1998)

74. Heptonstall, A., Barton, M., Cantley, C., Cumming, A., Cagnoli, G., Hough, J., Jones, R., Kumar, R., Martin, I., Rowan, S., Torrie, C., Zech, S.: Class. Quantum Grav. 27(3), 035013 (2010)

75. Plissi, M.V., Torrie, C.I., Husman, M.E., Robertson, N.A., Strain, K.A., Ward, H., Lück, H., Hough, J.: Rev. Sci. Instrum. 71(6), 2539 (2000)

76. Barr, B.W., Cagnoli, G., Casey, M.M., Clubley, D., Crooks, D.R.M., Danzmann, K., Elliffe, E.J., ler, S.G., Grant, A., Grote, H., Heptonstall, A., Hough, J., Jennrich, O., Lück, H., McIntosh, S.A., Newton, G.P., Palmer, D.A., Plissi, M.V., Robertson, D.I., Robertson, N.A., Rowan, S., Skeldon, 
K.D., Sneddon, P., Strain, K.A., Torrie, C.I., Ward, H., Willems, P.A., Willke, B., Winkler, W.: Class. Quantum Grav. 19(7), 1655 (2002)

77. Heptonstall, A., Cagnoli, G., Hough, J., Rowan, S.: Phys. Lett. A 354(5-6), 353 (2006)

78. Amico, P., Bosi, L., Carbone, L., Gammaitoni, L., Marchesoni, F., Punturo, M., Travasso, F., Vocca, H.: Rev. Sci. Instrum. 73(9), 3318 (2002)

79. Winkler, W., Danzmann, K., Rüdiger, A., Schilling, R.: Phys. Rev. A 44(11), 7022 (1991)

80. Strain, K.A., Danzmann, K., Mizuno, J., Nelson, P.G., Rdiger, A., Schilling, R., Winkler, W.: Phys. Lett. A 194(1-2), 124 (1994)

81. Lawrence, R.C.: Active wavefront correction in laser interferometric gravitational wave detectors. Ph.D. thesis, MIT, Boston (2003)

82. Lück, H., Freise, A., Goßler, S., Hild, S., Kawabe, K., Danzmann, K.: Class. Quantum Grav. 21(5), S985 (2004)

83. Ballmer, S., Frolov, V., Lawrence, R., Kells, W., Moreno, G., Mason, K., Ottaway, D., Smith, M., Vorvick, C., Willems, P., Zucker, M.: Thermal compensation system description. LIGO Document Center, LIGO-T050064-00-R (2005). http://dcc.ligo.org/

84. Kamp, C.J., Kawamura, H., Passaquieti, R., DeSalvo, R.: Nucl. Instru. Methods Phys. Res. A 607(3), 530 (2009)

85. Puppo, P., Ricci, F.: Cryogenics and Einstein Telescope (2010, this issue)

86. Nawrodt, R., Cunningham, L., Flaminio, R., Franc, J., Heinert, D., Hough, J., Martin, I., Morgado, N., Rowan, S., Schwarz, C., Seidel, P.: Mirror thermal noise calculation for ET. ET document ET-027-09 (2009). http://www.et-gw.eu/

87. Braginsky, V.B., Mitrofanov, V.P., Panov, V.I.: Systems with Small Dissipation. University of Chicago Press, Chicago (1985)

88. Ju, L., Notcutt, M., Blair, D., Bondu, F., Zhao, C.N.: Phys. Lett. A 218, 197 (1996)

89. Rowan, S., Byer, R.L., Fejer, M.M., Route, R., Cagnoli, G., Crooks, D.R.M., Hough, J., Sneddon, P.H., Winkler, W.: Test mass materials for a new generation of gravitational wave detectors. In: Proceedings of SPIE, vol. 4856 (2003)

90. Loriette, V., Boccara, C.: Appl. Opt. 42(4), 649 (2003)

91. White, G.K.: J. Phys. D Appl. Phys. 6(17), 2070 (1973)

92. Bilenko, I.A., Braginsky, V.B., Markova, N.Y.: Class. Quantum Grav. 19(7), 2035 (2002)

93. Mitrofanov, V.P., Tokmakov, K.V.: Phys. Lett. A 308(2-3), 212 (2003)

94. Ageev, A., Palmer, B.C., Felice, A.D., Penn, S.D., Saulson, P.R.: Class. Quantum Grav. 21(16), 3887 (2004)

95. McSkimin, H.J.: J. Appl. Phys. 24(8), 988 (1953)

96. Fine, M.E., van Duyne, H., Kenney, N.T.: J. Appl. Phys. 25(3), 402 (1954)

97. Strakna, R.E.: Phys. Rev. 123(6), 2020 (1961)

98. Travasso, F., Bosi, L., Dari, A., Gammaitoni, L., Vocca, H., Marchesoni, F.: Mater. Sci. Eng. A 521522, 268 (2009)

99. Wiedersich, J., Adichtchev, S.V., Rössler, E.: Phys. Rev. Lett. 84(12), 2718 (2000)

100. Travasso, F., Amico, P., Bosi, L., Cottone, F., Dari, A., Gammaitoni, L., Vocca, H., Marchesoni, F.: Europhys. Lett. 80(5), 50008 (2007)

101. Anderson, O.L., Bömmel, H.E.: J. Am. Ceram. Soc. 38(4), 125 (1955)

102. Amico, P., Bosi, L., Carbone, L., Gammaitoni, L., Marchesoni, F., Punturo, M., Travasso, F., Vocca, H.: Rev. Sci. Instrum. 73(1), 179 (2002)

103. Amico, P., Bosi, L., Carbone, L., Gammaitoni, L., Punturo, M., Travasso, F., Vocca, H.: Class. Quantum Grav. 19(7), 1663 (2002)

104. Cagnoli, G.: J. Phys. Conf. Ser. 32(1), 294 (2006)

105. Yamamoto, K., Miyoki, S., Uchiyama, T., Ishitsuka, H., Ohashi, M., Kuroda, K., Tomaru, T., Sato, N., Suzuki, T., Haruyama, T., Yamamoto, A., Shintomi, T., Numata, K., Waseda, K., Ito, K., Watanabe, K.: Class. Quantum Grav. 21(5), S1075 (2004)

106. Liberman, V., Bloomstein, T.M., Rothschild, M., Sedlacek, J.H.C., Uttaro, R.S., Bates, A.K., Peski, C.V., Orvek, K.: Materials issues for optical components and photomasks in $157 \mathrm{~nm}$ lithography. Papers from the 43rd international conference on electron, ion, and photon beam technology and nanofabrication (1999)

107. Yan, Z., Ju, L., Gras, S., Zhao, C., Blair, D.G.: Eur. Phys. J. Appl. Phys. 30, 189 (2005)

108. Nawrodt, R., Zimmer, A., Koettig, T., Nietzsche, S., Thuerk, M., Vodel, W., Seidel, P.: Eur. Phys. J. Appl. Phys. 38, 53 (2007) 
109. Numata, K., Bianc, G.B., Ohishi, N., Sekiya, A., Otsuka, S., Kawabe, K., Ando, M., Tsubono, K.: Phys. Lett. A 276(1-4), 37 (2000)

110. Numata, K., Bianc, G.B., Tanaka, M., Otsuka, S., Kawabe, K., Ando, M., Tsubono, K.: Phys. Lett. A 284(4-5), 162 (2001)

111. Rowan, S., Cagnoli, G., Sneddon, P., Hough, J., Route, R., Gustafson, E.K., Fejer, M.M., Mitrofanov, V.: Phys. Lett. A 265(1-2), 5 (2000)

112. Kovalik, J., Saulson, P.R.: Rev. Sci. Instrum. 64(10), 2942 (1993)

113. Tomaru, T., Suzuki, T., Uchiyama, T., Yamamoto, A., Shintomi, T., Taylor, C.T., Yamamoto, K., Miyoki, S., Ohashi, M., Kuroda, K.: Phys. Lett. A 301(3-4), 215 (2002)

114. Hull, R.: Properties of Crystalline Silicon. The Institution of Engineering and Technology, UK (1999)

115. Wortman, J.J., Evans, R.A.: J. Appl. Phys. 36(1), 153 (1965)

116. Mihailovich, R.E., Parpia, J.M.: Phys. Rev. Lett. 68(20), 3052 (1992)

117. Reid, S., Cagnoli, G., Crooks, D., Hough, J., Murray, P., Rowan, S., Fejer, M., Route, R., Zappe, S.: Phys. Lett. A 351(4-5), 205 (2006)

118. Nawrodt, R., Zimmer, A., Koettig, T., Schwarz, C., Heinert, D., Hudl, M., Neubert, R., Thürk, M., Nietzsche, S., Vodel, W., Seidel, P., Tünnermann, A.: J. Phys. Conf. Ser. 122(1), 012008 (2008)

119. Shimbo, M., Furukawa, K., Fukuda, K., Tanzawa, K.: J. Appl. Phys. 60(8), 2987 (1986)

120. van Veggel, A.A., Scott, J., Skinner, D.A., Bezensk, B., Cunningham, W., Hough, J., Martin, I., Murray, P., Reid, S., Rowan, S.: Class. Quantum Grav. 26(17), 175007 (2009)

121. Flaminio, R., Mavalvala, N., McClelland, D., Mueller, G., Reitze, D.H., Schnabel, R., Strain, K.A., Willke, B.: Tools and toys for third generation detectors (2010, this issue)

122. Brückner, F., Clausnitzer, T., Burmeister, O., Friedrich, D., Kley, E.B., Danzmann, K., Tünnermann, A., Schnabel, R.: Opt. Lett. 33(3), 264 (2008)

123. Amico, P., Bosi, L., Gammaitoni, L., Losurdo, G., Machesoni, F., Mazzoni, M., Parisi, D., Punturo, M., Stranga, R., Toncelli, A., Tonelli, M., Travasso, F., Vetrano, F., Vocca, H.: Rev. Sci. Instrum. 77(4), 044502 (2006)

124. Hu, S.M.: J. Appl. Phys. 53(5), 3576 (1982)

125. Hallam, J., Chelkowski, S., Freise, A., Hild, S., Barr, B., Strain, K.A., Burmeister, O., Schnabel, R.: J. Opt. A 11(8), 085502 (2009)

126. D’Ambrosio, E., O’Shaugnessy, R., Thorne, K., Willems, P., Strigin, S., Vyatchanin, S.: Class. Quantum Grav. 21(5), S867 (2004)

127. Mours, B., Tournefier, E., Vinet, J.Y.: Class. Quantum Grav. 23(20), 5777 (2006)

128. Beyersdorf, P.T., Zappe, S., Fejer, M.M., Burkhardt, M.: Appl. Opt. 45(26), 6723 (2006)

129. Tarallo, M.G., Miller, J., Agresti, J., D’Ambrosio, E., DeSalvo, R., Forest, D., Lagrange, B., Mackowsky, J.M., Michel, C., Montorio, J.L., Morgado, N., Pinard, L., Remilleux, A., Simoni, B., Willems, P.: Appl. Opt. 46(26), 6648 (2007)

130. Kennedy, S.A., Szabo, M.J., Teslow, H., Porterfield, J.Z., Abraham, E.R.I.: Phys. Rev. A 66(4), 043801 (2002)

131. Avino, S., Calloni, E., Milano, L., Fiore, L.D., Rosa, R.D., Tierno, A.: Phys. Lett. A 355(4-5), 258 (2006)

132. Brückner, F., Friedrich, D., Clausnitzer, T., Burmeister, O., Britzger, M., Kley, E.B., Danzmann, K., Tünnermann, A., Schnabel, R.: Opt. Express 17(1), 163 (2009) 